\title{
Bulletin of the American Mathematical Society
}

This journal is the official organ of the Society. It reports official acts of the Society and the details of its meetings. It contains some of the officially invited addresses presented before the Society, reviews of advanced mathematical books, and a department of research announcements.

The subscription price is $\$ 12.00$ per annual volume of six numbers.

Invited Addresses offered for publication should be sent to MURRAy GerstenHABER, Department of Mathematics, University of Pennsylvania, Philadelphia, Pennsylvania 19104.

Book Reviews should be sent to Gian-Carlo Rota, Mathematics Department, Massachusetts Institute of Technology, Cambridge, Massachusetts 02139.

All Research Announcements should be sent to M. H. ProtTer, Department of Mathematics, University of California, Berkeley, California 94720.

All other communications to the editors should be sent to the Managing Editor, MURRAy Gerstenhaber, at the above address.

\section{Proceedings of the American Mathematical Society}

This journal is devoted entirely to research in pure and applied mathematics and is devoted principally to the publication of original papers of moderate length. A department called Shorter Notes was established for the purpose of publishing very short papers of an unusually elegant and polished character, for which there is normally no other outlet.

Papers in algebra, number theory, and algebraic geometry should be sent to Grorge B. Seligman, Department of Mathematics, Yale University, New Haven, Connecticut 06520 or Joseph J. Rotman, Department of Mathematics, University of Illinois, Urbana, Illinois 61801 ; in modern or classical analysis to IRvING GLICKsBERG, Department of Mathematics, University of Washington, Seattle, Washington 98105, W. H. J. FucHs, White Hall, Cornell University, Ithaca, New York 14850 or to Allen Shields, Department of Mathematics, University of Michigan, Ann Arbor, Michigan 48104; in set-theoretic and general topology to ERNEST A. MichaEL, Department of Mathematics, University of Washington, Seattle, Washington 98105; in algebraic topology and all other geometry to P. EMERY THOMAs, Department of Mathematics, University of California, Berkeley, California 94720; in applied mathematics, differential equations, and related areas of analysis to WoLFGANG WAsOW, Mathematics Department, University of Wisconsin, Madison, Wisconsin 53706; in probability, statistics, and related fields to JoseuA Chover, Mathematics Department, University of Wisconsin, Madison, Wisconsin 53706; in logic, set theory, and related areas to W. W. BooNE, Mathematics Department, University of Illinois, Urbana, Illinois 61801 . All other communications should be addressed to the Managing Editor, W. H. J. Fuchs.

\section{Transactions of the American Mathematical Society}

This journal is devoted entirely to research in pure and applied mathematics, and includes in general longer papers than the PROcEEDINGS.

Papers in analysis and applied mathematics should be sent to J. J. KoHN, Department of Mathematics, Princeton University, Princeton, New Jersey 08540; in topology to Steve Armentrout, Department of Mathematics, Pennsylvania State University, University Park, Pennsylvania 16802; in algebra, number theory, and logic to D. A. BuchsBaum, Department of Mathematics, Brandeis University, Waltham, Massachusetts 02154; in geometry and abstract analysis to SHLOMO STERNBERG, Department of Mathematics, Harvard University, Cambridge, Massachusetts 02138; in statistics and probability to H. P. McKEAN, Department of Mathematics, Rockefeller University, New York, New York 10021; in mathematical logic and foundations to DANA Scotr, Department of Mathematics, Princeton University, Princeton, New Jersey 08540. All other communications to the editors should be addressed to the Managing Editor, DANa ScotT.

\section{Memoirs of the American Mathematical Society}

The Memorrs of the American Mathematical Society constitute a series of paperbound research tracts which are of the same general character as the papers published in the TRANSACTIONS. An issue of the MEMoIrs contains either a single monograph or a group of cognate papers. The MEMorrs are printed by the photo-offset process. Information on preparation of camera copy and charges for publication may be obtained by writing to the Editorial Department of the American Mathematical Society. 


\section{Journals Published by the \\ American Mathematical Society}

\section{Soviet Mathematics-Doklady}

This bimonthly is a translation journal containing the entire pure mathematics section of the DokLady AKademi Nauk SSSR, the Reports of the Academy of Sciences of the USSR. The DOKLADY for a year contains about 500 articles, each about 4 pages long.

\section{Mathematical Reviews}

This journal is devoted to abstracts and reviews of the current mathematical literature of the world. Two volumes of MATHEMATICAL REVIEWS will be published in 1970, Volume 38, and Volume 39. Each volume will consist of 6 regular issues plus an index issue. In each regular issue the abstracts and reviews are grouped under subject headings. Publication began in 1940.

\section{Notices of the American Mathematical Society}

This journal announces the programs of the meetings of the Society. It carries the abstracts of all contributed papers presented at the meetings of the Society and publishes news items of interest to mathematical scientists.

All communications should be addressed to the Editor, P.O. Box 6248, Providence, Rhode Island 02904. News items and insertions for each issue must be in the hands of the editor on or before the deadline for the abstracts for the papers to be presented in the meetings announced in that issue. These deadlines are published regularly on the back of the title page.

\section{Mathematics of Computation}

A journal devoted to original papers in numerical analysis, the application of numerical methods and high-speed calculator devices, the computation of mathematical tables, the theory of high-speed calculating devices and other aids to computation. In addition it publishes reviews and notes in these and related fields.

Prospective publications should be addressed to the Editor, Professor Eugene Isaacson, Courant Institute of Mathematical Sciences, New York University, 251 Mercer Street, New York, New York 10012. The author may suggest the name of an editor for review of his paper.

\section{New Publications}

This quarterly journal announces new books and journals in all fields of higher mathematics, including a section on both forthcoming and recently published books. The information is acquired from several sources, such as pamphlets and catalogs received from publishers and book dealers from many parts of the world.

\section{Mathematics of the USSR-Izvestija}

This journal is a cover-to-cover translation of Izvestija AKademir Naur SSSR SERIJA MATEMATIČESKajA published bimonthly by the Academy of Sciences of the USSR. It is a journal of current research in all fields of pure mathematics. Starting with Volume 1, Number 1, January-February 1967, which corresponds to the Russian original Tom 31, Number 1, the translation is prepared by the Plenum Publishing Corporation with the cooperation of the American Mathematical Society, and is published bimonthly by the American Mathematical Society.

\section{Mathematics of the USSR-Sbornik}

This journal is a cover-to-cover translation of MAtematičeskiY SBornik (New Series), published monthly by the Moscow Mathematical Society and the Academy of Sciences of the USSR. It is a journal of current research in all fields of pure mathematics. The translation is published monthly starting with Volume 1, Number 1 January 1967, which corresponds to the Russian original Tom 72 (114), No. 1. 
Herbert Federer. The singular sets of area minimizing rectifiable currents with codimension one and of area minimizing flat chains modulo two with arbitrary codimension..............

P. Antonelli, D. Burghelea and P. J. Kahn. Gromoll groups, Diff $S^{n}$ and bilinear constructions of exotic spheres............

Janice Zemanek. On the semisimplicity of integral representation

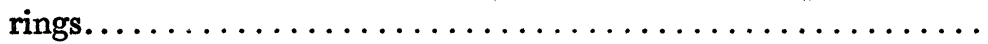

Martha Smith. On group algebras. .................

Nolan R. Wallach. Homogeneous positively pinched Riemannian

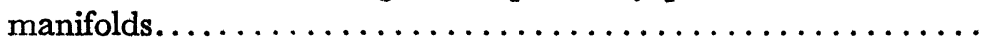

Julius L. Shaneson. Product formulas for $L_{n}(\pi) \ldots \ldots \ldots \ldots \ldots$

Anthony Phillips. Smooth maps transverse to a foliation........

Pol V. Lambert. On the minimum norm property of the Fourier projection in $L^{1}$-spaces and in spaces of continuous functions. . . . . .

S. K. Stein. $B$-sets and coloring problems. . . . . . . . . .

John R. Harper. On the cohomology of stable two stage Postnikov

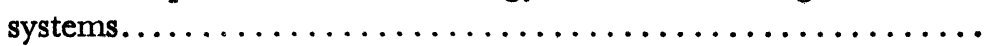

M. S. Ramanujan. Generalised nuclear maps in normed linear

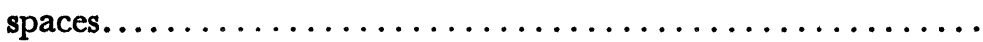

E. C. Zachmanoglou. Lie algebras of analytic vector fields and uniqueness in the Cauchy problem for first order partial differen-

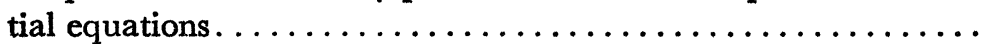

Lon Rosen. A $P(\phi)$ quantum field theory . . . . . . . . . . . .

David Wigner. Algebraic cohomology of topological groups.......

Melvyn S. Berger. On Morse theory and stationary states for non-

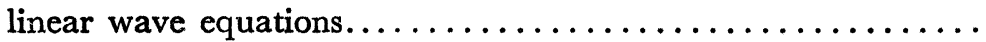

John Knopfmacher. Arithmetical properties of finite rings and algebras, and analytic number theory. . . . . . . . . . . .

Stewart B. Priddy. Koszul resolutions and the Steenrod algebra....

Martin H. Schultz. The condition number of a class of Rayleigh-

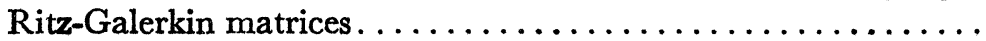

Bruce Calvert. Nonlinear evolution equations in Banach lattices...

Morton Curtis and Guido Mislin. Two new $H$-spaces. . . . . . . .

John T. Gannon and Arthur M. Jaffe. Relativistic covariance of an

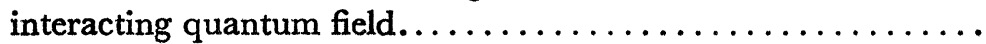

Bruce C. Berndt. On the average order of some arithmetical func-

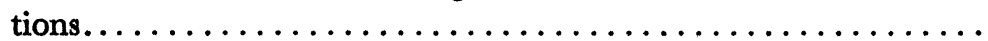

Norberto Kerzman. Hölder and $L^{p}$ estimates for solutions of $\bar{\partial} u=f$ in strongly pseudoconvex domains. . . . . . . . . . . .

Krishna B. Athreya and Samuel Karlin. Branching processes with random environments...................... 865

David Williams. Decomposing the Brownian path......... 871

A. Lawrence. Higher order compositions in the Adams spectral sequence.............................. 874

J. E. Humphreys. Modular representations of classical Lie algebras. 878

Séan Dineen. Holomorphic functions on a Banach space....... 883 


\section{CONTENTS}

\section{July, 1970}

Cathleen S. Morawetz. Energy flow: Wave motion and geometrical

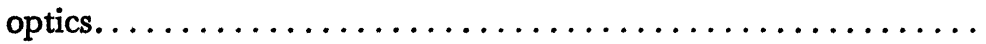

John Rhodes. Reviews of "The algebraic theory of semigroups," by A. H. Clifford and G. P. Preston; "Semigroups" by E. S. Ljapin; "The theory of finitely generated commutative semigroups," by László Rédei; "Elements of compact semigroups" by Karl Heinrich Hofmann and Paul S. Mostert.................

Elliott Lieb. Review of "Statistical Mechanics" by David Ruelle...

J. L. Doob. Review of "Markov Processes with Stationary Transition Probabilities" by Kai Lai Chung . . . . . . . . . . . . . .

The March Meeting in New York. . . . . . . . . . . . . .

Arthur Mattuck. Arthur Byron Coble. . . . . . . . . . . . .

Felix E. Browder, Djairo G. de Figueiredo and Chaitan P. Gupta. Maximal monotone operators and nonlinear integral equations of

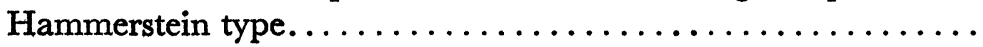

M. Csörgö and S. Csörgö. An invariance principle for the empirical process with random sample size.................

E. F. Beckenbach and T. A. Cootz. The second fundamental theorem for meromorphic minimal surfaces. . . . . . . . . . . . .

Weishu Shih. Une remarque sur la formule de residus. . . . . . . . .

C. E. Harle. Rigidity of hypersurfaces of constant scalar curvature. .

C. D. Feustel. On pasting balls to handlebodies...........

J. W. Robbin. On structural stability................

Norman Levitt. Generalized Thom spectra and transversality for

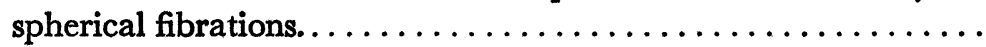

Alan McIntosh. Hermitian bilinear forms which are not semi-

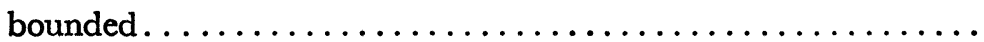

Albert Nijenhuis and Herbert S. Wilf. On a conjecture in the theory of permanents.

Charles D. Bass and Robert J. Daverman. A self-universal crumpled cube which is not universal. . . . . . . . . . . . . 740

William Smoke. Dimension and multiplicity for graded algebras... 743

D. G. Bourgin. On the Vietoris-Begle theorem............

Joanne Elliott and Martin R. Silverstein. On boundary conditions for symmetric submarkovian resolvents. . . . . . . . . . .

Naresh C. Jain and William E. Pruitt. The central limit theorem for the range of transient random walk.

Alan B. Poritz. On parallelism in Riemannian manifolds. .........

Richard L. W. Brown. Imbeddings, immersions, and cobordism of

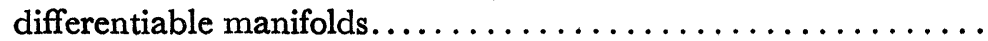

